\title{
Polarised photoelectrons from silver atoms
}

\author{
U Heinzmann, A Wolcke and J Kessler \\ Physikalisches Institut der Universität Münster, 4400 Münster, West Germany
}

Received 17 March 1980

\begin{abstract}
The polarisation of photoelectrons ejected by circularly polarised VUV radiation from silver atoms has been measured in the wavelength range between 150 and $160 \mathrm{~nm}$. Autoionisation states give rise to pronounced resonance structures in the photoionisation cross section and in the photoelectron polarisation. The measured polarisation yields direct information on the angular momentum quantum numbers $J$ of the autoionising states and on extrema of the cross section resonances.
\end{abstract}

\section{Introduction}

Fano predicted in 1969 that photoelectrons emitted by alkali atoms which are exposed to circularly polarised light should be polarised (Fano 1969a, b). This effect has been studied by several experimental and theoretical groups in the past few years and can now be said to be quantitatively understood (Kessler 1976 and references therein). Like the alkalis, a silver atom has one electron in the outermost shell, but its photoionisation cross section has a pronounced structure not far from threshold due to excitation of an electron from the next deeper shell. Such autoionising resonances affect the electron polarisation considerably, giving rise to a more complicated structure of the polarisation curve than in the case of the alkalis. Free silver atoms are therefore a proper sample for studying, on a one-electron system, the influence of autoionisation resonances on the Fano effect. This paper shows what information can be derived from such investigations. It also describes the high-temperature oven for producing the silver atomic beam.

\section{Theoretical description of the polarisation of photoelectrons ejected from silver atoms}

The ground state of Ag has the configuration $4 d^{10} 5 s\left({ }^{2} S_{1 / 2}\right)$, which is similar to that of the alkali atoms. Besides the excited-state configuration $4 \mathrm{~d}^{10} n \mathrm{p}$ with the ionisation threshold at $7.57 \mathrm{eV}(163.7 \mathrm{~nm})$ resulting from transitions of the $5 \mathrm{~s}$ electron, there are also the more complex configurations $4 \mathrm{~d}^{9} 5 \mathrm{~s} n^{\prime} x$ with ionisation thresholds near $12 \mathrm{eV}$ (about $100 \mathrm{~nm}$ ) (Johannsen and Lincke 1975).

When a photon of an energy between $7.57 \mathrm{eV}$ and $12 \mathrm{eV}$ is absorbed by the atom, the outer 5 s electron may obtain an energy exceeding the ionisation threshold, so that a continuum state is excited. If, however, the photon is absorbed by a $4 \mathrm{~d}$ electron, then atomic states of discrete energies are excited. The interaction of these discrete states with the continuum states of the same quantum numbers $J$ and $M_{J}$ and the same parity 
gives rise to autoionisation resonances, i.e. pronounced structures in the cross section and in the photoelectron polarisation, as discussed later in this paper. In table 1 the autoionising states of the silver atom between 150 and $160 \mathrm{~nm}$ are listed.

When circularly polarised $\sigma^{+}$light is used for photoionisation the selection rules $\left(\Delta J=0, \pm 1, \Delta L= \pm 1\right.$ and $\Delta M_{J}=+1$ ) allow transitions from the ground state ${ }^{2} \mathrm{~S}_{1 / 2}$ to the continuum states $\epsilon^{2} \mathrm{P}_{1 / 2}$ and $\epsilon^{2} \mathrm{P}_{3 / 2}$ which are degenerate in energy. Figure 1 illustrates the transitions produced by circularly polarised $\sigma^{+}$light for two different energies $E_{1}$ (transition 1-3) and $E_{2}$ (transition 4-6). In the figure we have used our earlier notation (Heinzmann et al 1970, 1975) indicating that the angular parts of the wavefunctions are proportional to linear combinations of two kets $\left|M_{S}, M_{L}\right\rangle$. The spin polarisation of a specific final state $\epsilon^{2} \mathrm{P}_{1 / 2}$ follows from the Clebsch-Gordan coefficients appearing in the wavefunctions. For $\epsilon^{2} \mathrm{P}_{1 / 2}\left(M_{J}=\frac{1}{2}\right)$ one obtains for the photoelectron polarisation averaged over all direction of emission $\bar{P}=\left(\frac{1}{3}-\frac{2}{3}\right) /\left(\frac{1}{3}+\frac{2}{3}\right)=-\frac{1}{3}=-33 \%$. For

Table 1. Autoionising states of silver between 150 and $160 \mathrm{~nm}$.

\begin{tabular}{|c|c|c|c|c|}
\hline \multicolumn{2}{|c|}{ Wavelengths } & \multirow[b]{2}{*}{$\begin{array}{l}\text { Identification of } \\
\text { the autoionising } \\
\text { states (Connerade } \\
\text { and Baig 1979) }\end{array}$} & \multirow[b]{2}{*}{$\begin{array}{l}\text { Polarisation } \\
\bar{P}(\%)\end{array}$} & \multirow[b]{2}{*}{$\begin{array}{l}\text { Cross section } \\
\text { (arbitrary units) } \\
\text { (Tegeder and Lincke } \\
\text { 1971) }\end{array}$} \\
\hline $\begin{array}{l}\text { Experimental data } \\
\text { (nm) } \\
\text { (Tegeder and } \\
\text { Lincke 1971) }\end{array}$ & $\begin{array}{l}\text { Calculated values } \\
\text { (nm) } \\
\text { (Connerade and } \\
\text { Baig 1979) }\end{array}$ & & & \\
\hline $157 \cdot 40$ & $157 \cdot 11$ & ${ }^{2} \mathrm{D}_{3 / 2}$ & +83 & 1 \\
\hline $154 \cdot 85$ & 155.82 & ${ }^{2} \mathrm{P}_{1 / 2}$ & -33 & 30 \\
\hline $151 \cdot 55$ & $152 \cdot 15$ & ${ }^{2} \mathrm{P}_{3 / 2}$ & +83 & 100 \\
\hline & 151.94 & ${ }^{4} D_{1 / 2}$ & -33 & 100 \\
\hline $150 \cdot 75$ & $150 \cdot 48$ & ${ }^{4} D_{3 / 2}$ & +83 & 50 \\
\hline
\end{tabular}

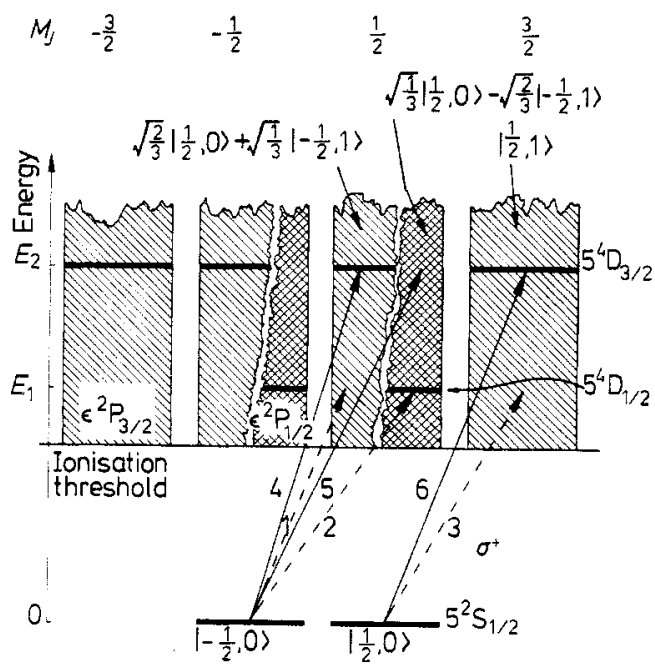

Figure 1. Level diagram of silver atom for photoionisation with circularly polarised $\sigma^{+}$ light. The transitions into the continuum and the autoionising states are shown for two energies $\left(E_{1}\right.$ and $\left.E_{2}\right)$. The angular parts of the wavefunctions are characterised by combinations of two kets $\left|M_{S}, M_{L}\right\rangle$. 
$\epsilon^{2} \mathrm{P}_{3 / 2}\left(M_{J}=\frac{1}{2}\right.$ and $\left.\frac{3}{2}\right)$ one obtains $\bar{P}=33 \%$ and $\bar{P}=100 \%$, respectively. Taking into account the different probabilities for transitions into $M_{J}=\frac{1}{2}$ and $M_{J}=\frac{3}{2}$ and assuming that the two magnetic sublevels of the ground state are equally populated (unpolarised atomic beam) one obtains an overall polarisation of $83 \%$ for the $\epsilon^{2} \mathrm{P}_{3 / 2}$ photoelectrons. The direction of the polarisation averaged over all directions of emission of the photoelectrons is parallel (positive polarisation) or antiparallel (negative polarisation) to the direction of the photon spins in the light beam.

According to Fano's theory, the polarisation $\bar{P}$ of the photoelectrons which are simultaneously produced in the $\epsilon^{2} \mathrm{P}_{1 / 2}$ and $\epsilon^{2} \mathrm{P}_{3 / 2}$ continuum states is given by

$$
\vec{P}=\frac{\frac{5}{6}\left(6\left|R_{3}\right|^{2}\right)-\frac{1}{3}\left(3\left|R_{1}\right|^{2}\right)-4\left|R_{1} \| R_{3}\right| \cos \delta}{6\left|R_{3}\right|^{2}+3\left|R_{1}\right|^{2}}
$$

where $R_{1}=\left|R_{1}\right| \exp \left(\mathrm{i} \delta_{1}\right)$ and $R_{3}=\left|R_{3}\right| \exp \left(\mathrm{i} \delta_{3}\right)$ are the radial matrix elements for transitions into $\epsilon^{2} \mathrm{P}_{1 / 2}$ and $\epsilon^{2} \mathrm{P}_{3 / 2}$. This formula, which is also valid for caesium where the phase difference $\delta=\delta_{1}-\delta_{3} \approx 0 \cdot 1$ is very small (Fano 1969b), can also be written in the form

$$
\bar{P}=\frac{\bar{P}\left(\epsilon^{2} \mathrm{P}_{3 / 2}\right) Q_{3 / 2}+\bar{P}\left(\epsilon^{2} \mathrm{P}_{1 / 2}\right) Q_{1 / 2}-I}{Q_{3 / 2}+Q_{1 / 2}}
$$

where $\bar{P}\left(\epsilon^{2} \mathrm{P}_{1 / 2}\right)=-\frac{1}{3}=-33 \%$ and $\bar{P}\left(\epsilon^{2} \mathrm{P}_{3 / 2}\right)=\frac{5}{6}=+83 \%$ are the polarisation values of the two continuum states and $Q_{1 / 2} \propto 3\left|R_{1}\right|^{2}$ and $Q_{3 / 2} \propto 6\left|R_{3}\right|^{2}$ are the partial cross sections for transitions into the two continua. $I=4\left|R_{1}\right|\left|R_{3}\right| \cos \delta$ describes the interference of the final continuum states $\epsilon^{2} \mathrm{P}_{1 / 2}$ and $\epsilon^{2} \mathrm{P}_{3 / 2}$. This term can be neglected in equation (2) if one of the matrix elements $R_{1}$ or $R_{3}$ is negligible compared with the other.

Let us now consider the influence of autoionising resonances such as the autoionising states $5^{4} \mathrm{D}_{3 / 2}$ and $5^{4} \mathrm{D}_{1 / 2}$ which have been indicated in figure 1 . The autoionising states can couple with continuum states of the same quantum numbers $J$ and $M_{J}$. This coupling has a strong effect on the energy dependence of the photoionisation cross section $Q$ as has been derived by Fano (1961). For example, the cross section of a ${ }^{2} P_{1 / 2}$ resonance is plotted qualitatively in figure 2(a) according to equation (21) of Fano's paper. The dotted line shows the cross section without the influence of autoionisation.

The behaviour of the polarisation in the region of that resonance is shown qualitatively in figure $2(b)$. It is typical for the polarisation curve to have a maximum, a zero crossing and a minimum. The polarisation can easily be calculated from equation (2) if the interference term $I$ is neglected. This is a good approximation at those energies where the cross section has a minimum or a maximum so that either the transition probability to $\epsilon^{2} \mathrm{P}_{1 / 2}$ or that to $\epsilon^{2} \mathrm{P}_{3 / 2}$ is negligible. In the case of an autoionising state with $J=\frac{1}{2}$ the electrons of the $\epsilon^{2} \mathrm{P}_{1 / 2}$ continuum $(\bar{P}=-33 \%)$ prevail in the maximum of the cross section $Q_{1 / 2}$. On the other hand, these electrons are missing in the minimum of $Q_{1 / 2}$ so that the $\epsilon^{2} \mathrm{P}_{3 / 2}$ continuum $(\bar{P}=+83 \%)$ determines the electron polarisation there. In the case of an autoionisation state with $J=\frac{3}{2}$ the roles of the two continua are interchanged. The polarisation curve in figure 2 is very schematic because the influence of the interference term $I$ has been ignored and because the cross sections without the influence of autoionisation have been assumed to be independent of energy (horizontal lines in figure $2(a)$ ). The superposition of the two continua described by the term $I$ does not show any effect in the cross section (which is given by the denominators of equations (1) and (2)), but it may affect the polarisation substantially. 


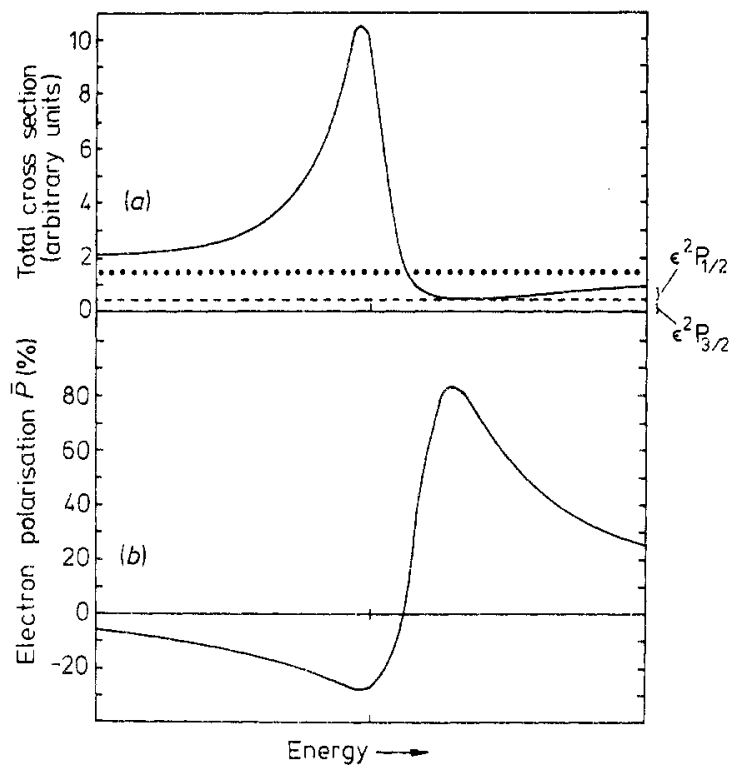

Figure 2. Qualitative behaviour of an autoionisation resonance: ( $a$ ) Energy dependence of the cross section disturbed by an autoionising state with $J=\frac{1}{2}$. The broken line gives the contribution of the undisturbed $\epsilon^{2} P_{3 / 2}$ continuum. The dotted line shows the cross section without the influence of autoionisation. (b) Photoelectron polarisation calculated from cross section (a) according to formula (2) with $I=0$.

\section{Apparatus}

\subsection{Experimental set-up}

A schematic diagram of the experimental set-up is shown in figure 3. Except for the atomic beam oven, all details of this apparatus have been described elsewhere (Heinzmann 1977,1978 ). The vUV radiation, produced by an $\mathrm{H}_{2}$ discharge lamp, passes through a Seya-Namioka vuv monochromator (McPherson model 235, $0.5 \mathrm{~m}$ ) and is linearly polarised by an $\mathrm{MgF}_{2}$ double Sénarmont prism. An $\mathrm{MgF}_{2}$ quarter-wave double plate (at $150 \mathrm{~nm}$ ) then circularly polarises the radiation which is finally focused onto the silver atomic beam. The photoelectrons which are produced within an electrostatic quadrupole field are extracted regardless of their direction of emission, focused by electron optical lenses and accelerated to $120 \mathrm{keV}$ for polarisation analysis in a Mott detector (gold foil $180 \mu \mathrm{g} \mathrm{cm}^{-2}$, Sherman function $S=-0 \cdot 26 \pm 0 \cdot 01$ ). During the polarisation measurement the light polarisation is changed from $\sigma^{-}$to $\sigma^{+}$and vice versa every minute in order to eliminate instrumental asymmetries (Heinzmann 1978). The light polarisation is analysed by a second $\mathrm{MgF}_{2}$ prism and the light intensity is detected by a photomultiplier tube with a sodium salicylate layer.

\subsection{Atomic beam oven}

The high-temperature oven for evaporation of silver is shown in figure 4. The crucible was made of boron nitride (BN, Cerac Inc., Milwaukee, Wis., USA) and was carried by a Stenan plate (Rosenthal Stemag GmbH, Lauf, Germany) which was held by four 


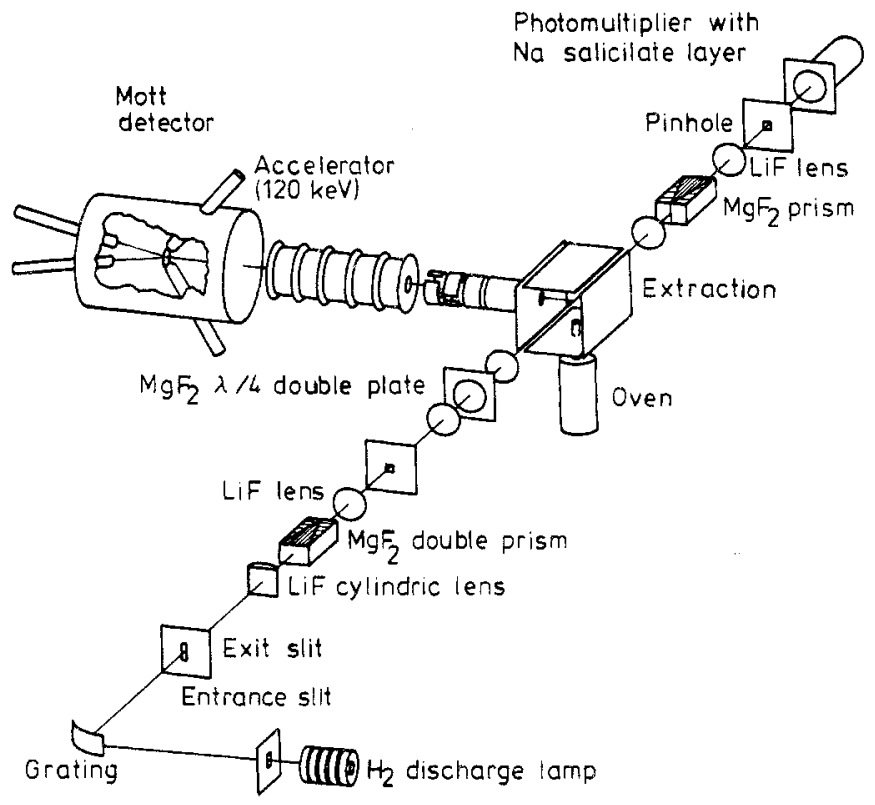

Figure 3. Schematic diagram of the apparatus.

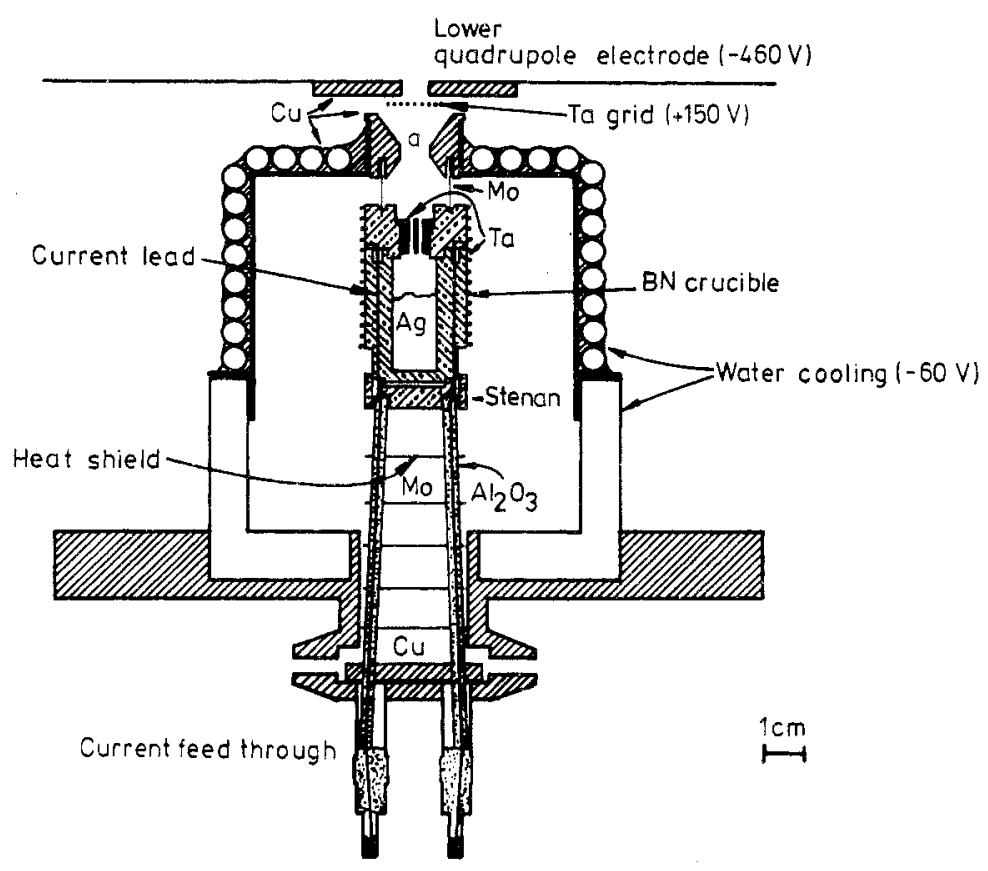

Figure 4. Cross sectional view of the high-temperature oven used to produce the silver vapour beam.

$\mathrm{Al}_{2} \mathrm{O}_{3}$ tubes. These tubes also contained the wires for heating the crucible. As a heater a tantalum wire of $0.4 \mathrm{~mm}$ diameter and $2.80 \mathrm{~m}$ length was wound as a bifilar coil onto the crucible into which a double thread had been grooved. The magnetic field produced 
at the target by the heating coil was smaller than $0.08 \mathrm{~A} \mathrm{~m}^{-1}$ when the oven was heated with a power of $1 \cdot 1 \mathrm{~kW}(\mathrm{AC})$. The Earth's magnetic field was reduced to below $0.8 \mathrm{~A} \mathrm{~m}^{-1}$. The crucible was surrounded by a water-cooled enclosure to prevent thermal radiation from the oven reaching the lower quadrupole electrode.

In order to prevent thermionic background electrons from reaching the detection system, the water cooling was connected to ground by a $4.7 \mathrm{M} \Omega$ resistor and thus became charged to about $-60 \mathrm{~V}$ by the thermionic emission current. In addition, a tantalum grid $(+150 \mathrm{~V})$ above the aperture (a) collected a great number of background electrons. In this way the background count rate has been reduced from 1.5 million to 50-100 electrons per minute as measured in the Mott detector. The count rate of the photoelectrons was six times higher. In the cap of the crucible there was a tantalum nozzle with seven channels, which directed the silver vapour beam to the aperture (a). This cold aperture emitted no thermionic electrons. It was funnel shaped to deform the electrostatic field in order to deflect background electrons.

The heating coil was divided into two separate coils. The cap of the crucible could thus be kept at a higher temperature than the lower part so that no silver could condense in the nozzle. The temperature $(1700 \mathrm{~K})$ was measured with a $\mathrm{Pt} \mathrm{Rh-Pt} \mathrm{thermocouple}$ dipped into the liquid silver. The thermocouple was embedded in refractory cement to avoid direct contact with the silver. The equilibrium of heating power and thermal radiation kept the temperature constant. At these temperatures the vapour pressure of silver is nearly $700 \mathrm{~Pa}$ (Rosebury 1965). The purity of the silver used was $99.999 \%$ (Koch-Light Lab. Ltd, England) and the fraction of undesired $\mathrm{Ag}_{2}$ molecules was less than $10^{-3}$ (Gmelin 1970).

\section{Results and discussion}

In figure 5(a) two intensity curves measured with different resolution are shown. In addition, the position of the autoionisation states (Tegeder and Lincke 1971) and their total angular momentum $J$ (Connerade and Baig 1979) is given. The wavelength scale is arranged so that the photon energy increases to the right. These measurements have been made by replacing the accelerator with a secondary electron multiplier (Channeltron, Valvo type B 419 BL-01).

The horizontal error bars give the bandwidths $\dagger$ of the radiation used $(0.25 \mathrm{~nm}$ and $1.5 \mathrm{~nm}$ bandwidths for the full and broken curves, respectively), while the vertical error bars show the root mean square of several measured curves. There is quite good agreement between the positions of the peaks measured and the values given in the literature. Even though with a bandwidth of $1.5 \mathrm{~nm}$ the resonances at $151.55 \mathrm{~nm}$ and $150.75 \mathrm{~nm}$ remained unresolved, this bandwidth has been used for the polarisation measurements because the rate of photoelectrons was then larger than with a bandwidth of $0.25 \mathrm{~nm}$ by a factor of 30-40. The measured polarisation is shown in figure $5(b)$. Each vertical error bar is the RMS sum of the errors of the light polarisation, the Sherman function, and the single statistical error.

\footnotetext{
$\left\lceil\right.$ Because the intensity distribution of the UV light produced by means of the $\mathrm{H}_{2}$ discharge lamp shows a many-line spectrum, the horizontal error bar is properly speaking the (half-width) resolution of.the monochromator. However, the density of lines in the many-line spectrum is so high (44 lines per nm), as pointed out by Herzberg and Howe (1959), that the lines merge into a quasicontinuum. Thus the horizontal error bars can be seen to be bandwidths.
} 


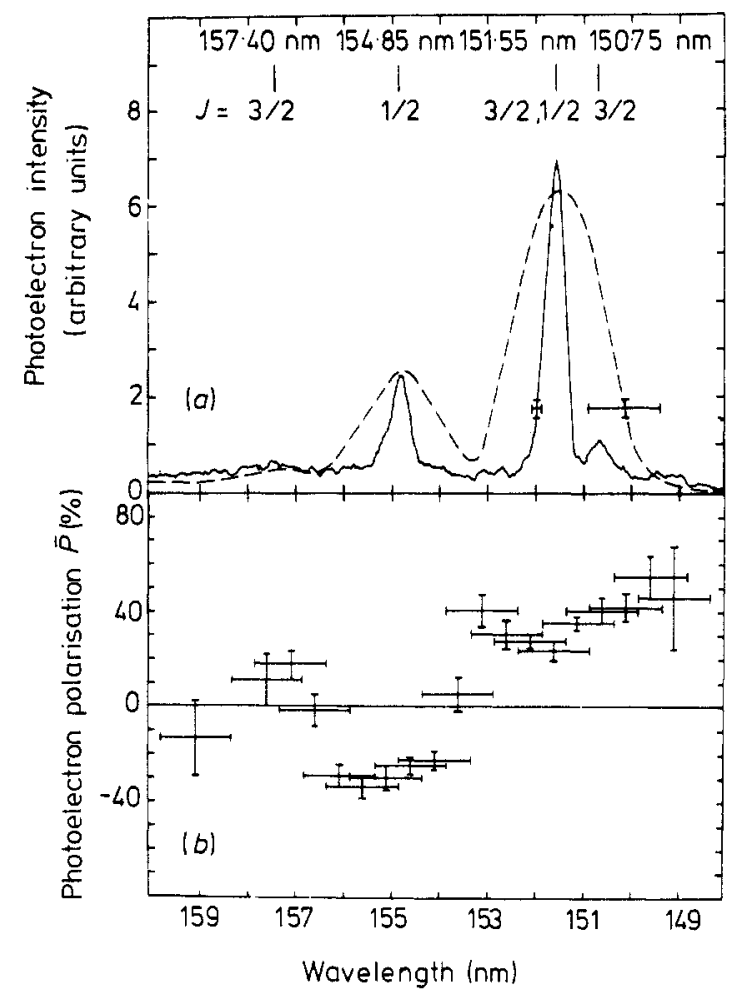

Figure 5. (a) Measured intensity of the photoelectrons and positions of the autoionising states (Tegeder and Lincke 1971) with angular momenta $J$ (Connerade and Baig 1979). (b) Measured photoelectron polarisation produced by circularly polarised light.

Near the transition at $154.85 \mathrm{~nm}$ the largest possible value of negative polarisation $(\bar{P}=-33 \%)$ is reached within the error limits. This corresponds to the situation depicted in figure 2 where, near the resonance maximum, the ${ }^{2} \mathrm{P}_{1 / 2}$ photoelectrons with $\bar{P}=-33 \%$ dominate. The polarisation measurements also indicate whether the minimum of the cross section resonance (figure $2(a)$ ) is located to the right or to the left of the maximum, information which the intensity curves do not yield even with $0.25 \mathrm{~nm}$ bandwidth. According to figure 2, the polarisation curve should go from negative to high positive values in the neighbourhood of the cross section minimum. This is seen in figure 5 to occur at the short-wavelength side of the intensity maximum, showing that the intensity minimum is located at this side. The fact that, compared with figure 2 , the minimum and the zero crossing of the polarisation are shifted to the left or right, respectively, is caused by the finite bandwidth, as has been found by convolution of ideal intensity curves like those shown in figure $2(a)$ with the bandwidth and the approximate intensity distribution of the radiation. The zero crossing at the long-wavelength side is caused by the resonance of $157.4 \mathrm{~nm}$.

Between $150 \mathrm{~nm}$ and $153 \mathrm{~nm}$ the polarisation values show a relative minimum $(23 \%)$ between two maxima $(40 \%$ and $54 \%)$. This gives evidence of an (unresolved) resonance peak of the $\epsilon^{2} \mathrm{P}_{1 / 2}$ continuum which is positioned between two resonance peaks of the $\epsilon^{2} \mathrm{P}_{3 / 2}$ continuum, a fact which is completely masked in the $1.5 \mathrm{~nm}$ intensity curve. The double peak at $151.55 \mathrm{~nm}$ (cf table 1) has not been resolved even in 
the intensity measurement with a $0.25 \mathrm{~nm}$ bandwidth. The polarisation indicates, however, that there are two peaks and it also verifies the sequence of their total angular momenta $J$ as a function of the wavelength predicted by theory (Connerade and Baig 1979).

In our discussion we have neglected the interference term $I$ in equation (2) because the experimental data are not accurate enough to find evidence for its influence. The present results are intended to show the possibilities of polarisation measurements. Despite the large bandwidth used, information like the sequence of the resonance maximum and the flat minimum or the sequence of closely neighbouring resonance peaks of different $J$ is obtained. Measurements with synchrotron radiation now being performed with other elements (Heinzmann et al 1979) would make possible investigations with much better resolution and accuracy and would allow a more detailed discussion of the autoionising resonances.

\section{Acknowledgments}

We are indebted to Dr B Cleff for helpful advice concerning the construction of the oven (Mühlen and Cleff 1978). We gratefully acknowledge support from the Deutsche Forschungsgemeinschaft.

\section{References}

Connerade J F and Baig M A 1979 Proc. R. Soc. A 365 253-65

Fano U 1961 Phys. Rev. 124 1866-78

- 1969a Phys. Rev. 178 131-6

1969b Phys. Rev. $\mathbf{1 8 4} 250-1$

Gmelin 1970 Handbuch der Anorganischen Chemie 61 (Weinheim: Verlag Chemie) p 250

Heinzmann U 1977 J. Phys. E: Sci. Instrum. $101001-5$

1978 J. Phys. B: Atom. Molec. Phys. 11 399--412

Heinzmann U, Heuer $H$ and Kessler J 1975 Phys. Rev. Lett. 34 441-4

1976 Phys. Rev. Leit. 36 1444-7

Heinzmann U, Kessler J and Lorenz J 1970 Z. Phys. 240 42-61

Heinzmann U, Schäfers F, Thimm K, Wolcke A and Kessler J 1979 J. Phys, B: Atom. Molec. Phys. 12 L679

Herzberg E and Howe L L 1959 Can. J. Phys. 37 636-59

Johannsen $\mathrm{H} U$ and Lincke R 1975 Z. Phys. A 272 147-54

Kessler J 1976 Polarized Electrons (Berlin: Springer) pp 123-46

Mühlen H J and Cleff B 1978 Nucl. Instrum. Meth. 154 193-4

Rosebury F 1965 Handbook of Electron Tube and Vacuum Techniques (Reading, Mass.: Addison-Wesley) p 143

Tegeder K and Lincke R 1971 Z. Phys. 247 51-9 